\title{
A Low-power Star-topology Body Area Network Controller for Periodic Data Monitoring Around and Inside the Human Body
}

\author{
Sungdae Choi, Seong-Jun Song, Kyomin Sohn, Hyejung Kim, Jooyoung Kim, Jerald Yoo and \\ Hoi-Jun Yoo \\ Korea Advanced Institute of Science and Technology, Daejeon, Korea \\ next@eeinfo.kaist.ac.kr
}

\begin{abstract}
More than hundred sensor devices are required to monitor various body signals. The body area network (BAN) connecting the sensor devices with the controller needs low power consumption and real-time operations, which is difficult to implement by conventional controller chips. In this paper, a low power controller chip is designed and fabricated to manage the proposed network with low power consumption. It periodically monitors the data from maximum 255 sensor devices with less than 2- $\mathrm{mW}$ power consumption. The controller is implemented in $25 \mathrm{~mm}^{2}$ silicon area and its operation is successfully demonstrated on the test system board.
\end{abstract}

\section{Introduction}

Technical development of the electronic devices has changed the placement of a personal computer from a desk to a lab and even to a human hand. And now the computer is embedded in a cloth or attached to a human body as a wearable computer so that it can provide a human-friendly computing environment and user-customized services. The wearable computer system can be used for various ways such as mobile healthcare monitoring [1], augmented reality system [2] as well as general PC purpose [3].

To realize such applications, a number of data should be collected to analyze the surroundings of the user such as the location, direction of the user's sight as well as the health conditions. Figure 1 shows an example of data to be collected for monitoring the health conditions and tracking the user position. Monitoring vital signs, for example, needs at least four bio signals including pulse, body temperature, a breathing rate and blood pressure. And it needs more than four sensors to catch more accurate data. The body temperature can be measured throughout the human body for precise measurement and it means that the system needs several temperature sensors. Such data should be collected periodically for continuous real-time monitoring and the system needs high performance to manage a lot of sensors and data.

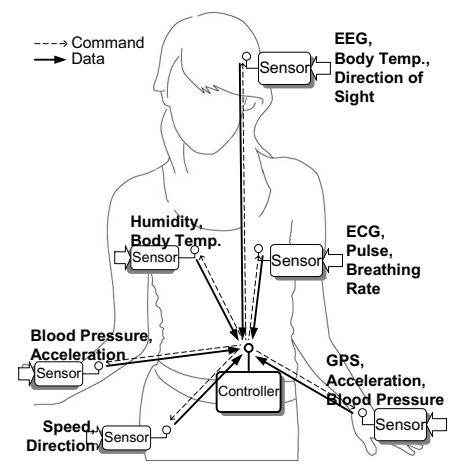

Figure 1. Body Area Network with Various Sensor Devices

In this paper we provide the low-power network platform to cover hundreds of sensor devices around the human body area and the implementation of the body area network (BAN) controller which manages up to 255 external sensor devices. Section 2 will describe the detailed controller and the implementation results will be shown in section 3. And we will make conclusion in section 4 .

\section{Star Network Controller}

Figure 2 shows the architecture of the proposed network controller with low-power consumption. It consists of a schedule director (SD) which generates periodic signals with low power consumption, a general-purpose 16bit RISC processor and several memory blocks. Node management is carried out independently by the SD by sending out data-requests to sensor devices and receiving data. This process is carried out without any processor involvement. For data-requests simple a packet structure is used so that most transceiver modules can be attached to the controller using minimum glue logic. The RISC works with high clock frequency to run generic programs providing high performance while SD runs at low clock to conserve energy. The low clock operation 
reduces unnecessary signal transition and low operation voltage, which bring low power operation.

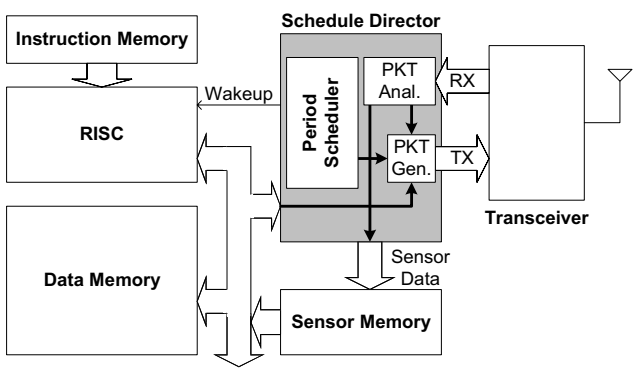

Figure 2. Proposed Controller Architecture

\section{System Implementation}

Figure 3 shows the test system to verify the proposed controller chip. The chip is fabricated by $0.18-\mu \mathrm{m}$ CMOS process and $25 \mathrm{~mm}^{2}$ silicon area. The proposed system requires small numbers of $\mathrm{I} / \mathrm{O}$ pins for small chip size and low cost. Although it is packaged by 256-pin QFP package, 228-pins are for the test purpose, 16-pins are unused and 12-pins are used for the normal operations. In the test system board, the FPGA reads the values of the control registers and the instruction codes from the flash memory and writes them to the controller chip. When it finishes dumping the program codes, the controller starts working by generating the data-request signals in accordance with the programmed schedule.

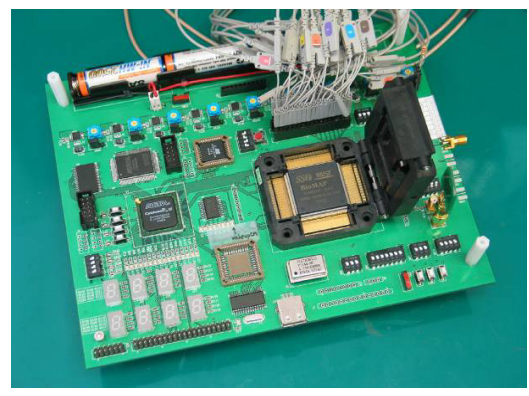

Figure 3. Test System Board

Figure 4 (a) shows the data-requests for four nodes with different periods. Figure 4 (b) depicts the waveforms of the detailed operations for each second. It shows that two nodes ID\#1 and ID\#252 are polled. The major signal transaction during the operation is just 8-bit counter operation for searching an ID number to be polled and it requires negligible power consumption. Once an ID is issued, SD transmits the request packet, waits for the reception packet and continues checking the next ID number. In the figure, checking 254 nodes are completed in $0.2 \mathrm{~s}$ and in the rest of the time, $0.8 \mathrm{~s}$, no signal transaction happens to prohibit unnecessary power consumption. Figure 4 (c) is the measured data transaction of one data request. The 1-second of real-time clock is generated by dividing the SD clock, $16.384-\mathrm{kHz}$ and the accumulated time error depends on the accuracy of SD clock.

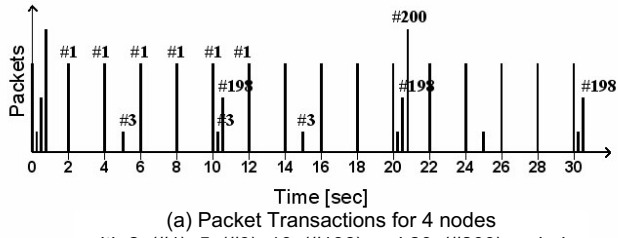
with 2s(\#1), 5s(\#3), 10s(\#198) and 20s(\#200) periods

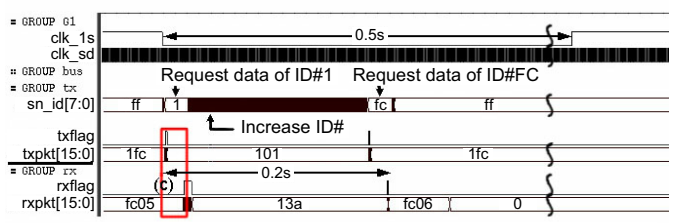

(b) Waveforms of the Packet Transaction for Data-Request

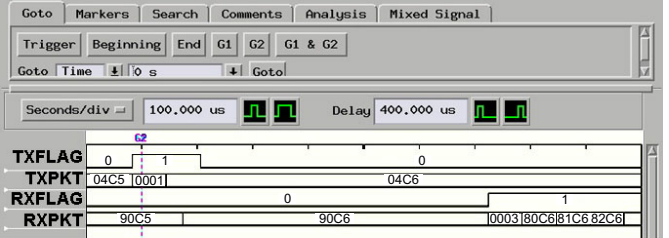

(c) Measured Packet Transactino by SD Operation

Figure 4. Periodic Data Request Operation

\section{Conclusions}

This paper proposes a centralized star network topology for low power BAN and implements a low power controller chip which is crucial for the proposed network system. With the node management by SD, the controller shows less than $2 \mathrm{~mW}$ power consumption for 255 node management. SD generates the periodic events for 255 sensor nodes with $0.8 \mathrm{~mm} \mathrm{x}$ $0.4 \mathrm{~mm}$ silicon area, which is about $20 \mathrm{x}$ size of conventional single event generation block. The $25 \mathrm{~mm}^{2}$ chip with $0.18-\mu \mathrm{m}$ CMOS process includes 16bit RISC, memory blocks as well as SD.

\section{References}

[1] D. Konstantas and R. Herzog, "Continuous monitoring of vital constants for mobile users: the MobiHealth approach," International Conference of the IEEE EMBS, pp.3728-3731, Sep 2003.

[2] Ryuhei Tenmoku, et al., "A Wearable Augmented Reality System Using Positioning Infrastructures and a Pedometer," IEEE ISWC, 2003

[3] Rich De Vaul, et al., "MIThril 2003: Applications and Architecture," IEEE ISWC, 2003 